\title{
Comparison of Forest Parameter Estimation Techniques Using SAR Data
}

\author{
Yunjin Kim and Jakob van Zyl \\ Jet Propulsion Laboratory \\ California Institute of Technology \\ 4800 Oak Grove Drive \\ Pasadena, CA 91109-8099 \\ Tel: (818) 354-9500 \\ Fax: (818) 353-5285 \\ E-mail: ykim@radar-sci.jpl.nasa.gov
}

\begin{abstract}
It is important to monitor forests in order to understand the impacts of global climate changes on terrestrial ecosystems. To characterize forest changes, it is useful to parameterize a forest using several parameters, such as biomass, basal area, tree density, tree height, and trunk diameter. These parameters are not independent and some of them are related by allometric equations. Remote sensing data can be used for estimating some forest parameters and others may be retrieved using allometric equations. Many researchers reported algorithms to estimate forest parameters using polarimetric SAR data. However, these algorithms cannot be applied to all types of forests without additional information on the forest type and environmental conditions since radar measurements depend on the tree structure, incidence angle, and environmental conditions. The backscattering cross section also saturates as forest parameters, such as biomass and the tree height, increase.
\end{abstract}

Forest parameters also have been estimated using SAR interferometry. Specifically, the interferometric correlation coefficient has been used to estimate the angular range of volume scattering. In this paper, we compare and contrast polarimetric and interferometric approaches to understand their advantages and limitations using NASA/JPL AIRSAR data.

\section{INTRODUCTION}

Many studies have been performed to estimate forest parameters using polarimetric SAR data [1,2]. Even though initial results of these studies were promising, it was found that forest parameter estimation techniques must solve several challenging problems. These problems are caused by the fact that radar return is sensitive to the different tree structure, environmental factors, and the radar imaging geometry. In addition, radar responses from forest areas saturate as biomass increases [3]. A successful algorithm must reduce the effects caused by these problems. If forest biomass is estimated and the forest type is known, tree heights and other forest parameters can be derived using allometric equations.

Recently, the interferometric correlation coefficient has been used for estimating tree heights [4,5]. The fundamental concept of this approach is that the interferometric correlation will decrease if more scattering objects are present in a pixel. A simple parameter to measure this decorrelation is the angular scattering range of a pixel to an interferometric antenna. However, in order to relate the tree height to the interferometric correlation coefficient accurately, one must assume a scattering profile within a pixel. Since several simple functions have been used to describe the scattering profile, it is necessary to understand the variation of estimated parameters for these different scattering profiles.

In this paper, we first discuss a method to retrieve biomass using polarimetric SAR data. Then, the interferometric correlation coefficient variation is studied for several backscattering cross section profiles. Finally, we conclude this paper by comparing these two techniques.

\section{FOREST PARAMETER ESTIMATION USING POLARIMETRIC SAR DATA}

The polarimetric parameters can be divided into two classes: absolute polarimetric parameter and relative polarimetric parameter. For example, the absolute polarimetric parameters are polarimetric backscattering cross sections and eigenvalues. The relative parameters include the $\mathrm{HH}$ and VV correlation coefficient, entropy, anisotropy, and the radar vegetation index. First, we will estimate biomass using polarimetric SAR data. Since polarimetric responses depend upon the tree structure, incidence angle, and environmental conditions, we need to develop a method to minimize the dependence of these three factors. Here, we show that the relative polarimetric 
parameters are less sensitive to both incidence angle and environmental condition effects. From [6], it was shown that $\sigma / \cos \theta_{i}$ would minimize the incidence angle $\left(\theta_{i}\right)$ dependence. Environmental changes such as precipitation and the freeze/thaw transition affect the dielectric constant of an imaged terrain. Therefore, polarimetric backscattering cross sections $\left(\sigma_{\text {pqrs }}\right)$ can be written as

$$
\sigma_{p q r s}=F(\varepsilon) \cos \theta_{i} I_{p q r s}
$$

where $F(\varepsilon)$ is a factor that represents environmental changes and $I_{p q r s}$ is a polarimetric measure that does not depend on the incidence angle and the environmental changes. This means that the relative polarimetric parameters are not very sensitive to the incidence angle and environmental changes. This can be shown by considering the radar vegetation index $(R V I)$ defined as

$$
R V I=\frac{8 \sigma_{h v h v}}{\sigma_{h h h h}+\sigma_{v v v v}+2 \sigma_{h v h v}}
$$

where $\sigma_{h v h v}$ is the cross-polarization backscattering cross section and $\sigma_{h h h h}$ and $\sigma_{v v v v}$ are co-polarization backscattering cross sections. These relative parameters are also insensitive to absolute calibration error.

The backscattering cross sections saturate as forest biomass increases. Therefore, these polarimetric algorithms are most suitable for monitoring the level of regrowth especially when time series data are available. We have observed that the relative polarimetric parameters saturate faster than the absolute polarimetric parameters. Therefore, we use the relative polarimetric parameters to derive a multiplicative factor in the absolute polarimetric parameters of different SAR data for biomass estimation.

Several theoretical investigations showed that the polarimetric responses from a forest could vary significantly depending upon the tree structure. In this paper, we examine a classification technique based on the strength of three dominant scattering mechanisms: surface scattering, double bounce scattering, and randomly oriented thin cylinders scattering. Their polarimetric responses are shown in the following six equations:

Surface scattering conditions:

$$
\begin{aligned}
& \operatorname{Re}\left(\sigma_{h h v v}\right)>\sigma_{h v h v} \\
& \sigma_{v v v v}>\sigma_{h h h h}
\end{aligned}
$$

Double bounce scattering conditions:

$$
-\operatorname{Re}\left(\sigma_{h h v}\right)>\sigma_{h v h v}
$$

$$
\sigma_{v v v v}<\sigma_{h h h h}
$$

Randomly oriented thin cylinders scattering conditions:

$$
\begin{aligned}
& \sigma_{v v v v}=\sigma_{h h h h} \\
& \sigma_{h h v v}=\sigma_{h v h v}=\frac{1}{3} \sigma_{h h h}
\end{aligned}
$$

A classification result will be presented to demonstrate the effectiveness of the technique.

\section{TREE HEIHT ESTIMATION USING INTERFEROMETRIC SAR DATA}

The interferometric correlation coefficient $(\gamma)$ is defined as

$$
\gamma=\frac{\left\langle\psi_{1} \psi_{2}^{*}\right\rangle}{\sqrt{\left\langle\psi_{1} \psi_{1}^{*}\right\rangle\left\langle\psi_{2} \psi_{2}^{*}\right\rangle}}
$$

where $\psi_{1}$ and $\psi_{2}$ are electromagnetic fields measured at two interferometric channels. For single pass interferometric SAR, the interferometric correlation coefficient can be written as

$$
\gamma=\gamma_{S c a t} \gamma_{S N R}
$$

where $\gamma_{\text {Scat }}$ is the correlation coefficient due to the scattering geometry and $\gamma_{S N R}$ is determined by the radar SNR (Signal to Noise Ratio). It is important to separate these two correlation components to isolate $\gamma_{\text {Scat }}$ that contains the information on the scattering geometry.

In order to relate $\gamma_{\text {Scat }}$ to the physical scattering geometry, a scattering profile function must be assumed and the relationship [4] is given by

$\gamma_{\text {scat }}=\frac{\iiint \sigma_{p q r s}(x, y, z) F e^{-i k\left(r_{1}-r_{2}\right)} d x d y d z}{\iiint \sigma_{p q r s}(x, y, z) F d x d y d z}$ 
where $\sigma_{\text {pqrs }}(x, y, z)$ is the backscattering profile, $F=F\left(x_{0}, x, r_{0}, r\right)$ is a two dimensional impulse response, $r_{0}=\left(y_{0}, z_{0}\right)$, and $r=(y, z)$. The slant range distances to two interferometric antennas are denoted by $r_{1}$ and $r_{2}$, respectively. In this paper, we use several scattering profiles to understand the interferometric correlation variation due to the scattering profile change.

\section{CONCLUSIONS}

In this paper, we studied two methods to estimate forest parameters. Using polarimetric SAR data, forest biomass and other forest parameters can be estimated. A new technique was introduced to reduce the environmental and incidence angle effects using relative polarimetric parameters. The interferometric correlation coefficient is sensitive to the scattering profile if one can remove the SNR effect. This relationship can be examined using various scattering profiles to understand the effectiveness of the method. Both techniques are promising in estimating forest parameters. However, more ground truth data are required to verify the accuracy of these techniques.

\section{ACKNOWLEDGMENT}

The research described in this paper was carried out at the Jet Propulsion Laboratory, California Institute of Technology, under a contract with the National Aeronautics and Space Administration.

\section{REFERENCES}

[1] T. Le Toan, A. Beaudoin, J. Riom, and D. Guyon, "Relating Forest Biomass to SAR Data, " IEEE Trans. Geosci. Remote Sens., Vol. 30, No. 2, pp. 403-411, 1992.

[2] M. C. Dobson, F. T. Ulaby, L. E. Pierce, T. L. Sharik, K. M. Bergen, J. Kellndorfer, J. R. Kendra, E. Li, Y. C. Lin, A. Nashashibi, K. Sarabandi, and P. Siqueira, "Estimation of Forest Biophysical Characteristics in Northern Michigan with SIR-C/XSAR," IEEE Trans. Geosci. Remote Sens., Vol. 33, No. 4, pp. 877-895, 1995.

[3] M. C. Imhoff, "Radar Backscatter and Biomass Saturation: Ramifications for Global Biomass Inventory," IEEE Trans. Geosci. Remote Sens., Vol. 33, No. 2, pp. 511-518, 1995.

[4] E. Rodriguez, T. R. Michel, and D. J. Harding, "Interferometric Measurement of Canopy Height Characteristics of Coniferous Forests," submitted for publication

[5] R. N. Treuhaft, S. N. Madsen, M. Moghaddam, and J. J. van Zyl, "Vegetation Characteristics and
Underlying Topography from Interferometric Radar," Radio Science, Vol. 31, No. 6, pp. 1449-1485, 1996.

[6] D. H. Hoekman and M. J. Quinones, "Land cover type and biomass classification using AIRSAR data for evaluation of monitoring scenarios in the Colombian Amazon," IEEE Trans. Geosci. Remote Sens., Vol. 38, No. 2, pp. 685-696, 2000. 